\title{
MYOSITIS AS A DISEASE MANIFESTATION OF CHRONIC RECURRENT MULTIFOCAL OSTEOMYELITIS: A CASE REPORT.
}

Vinicius Verlangieri Soubihe ${ }^{1, \star}$, Diego de Paula Ferreira Nunes ${ }^{1}$, Raíssa Dudienas Domingues Pereira ${ }^{1}$, Caio Rustichelli Cardoso ${ }^{1}$, Thaís Ferrarini Tavares ${ }^{1}$, Eliane Maria Ingrid Amstalden ${ }^{1}$, Michel Alexandre Yazbek ${ }^{1}$, Thiago Junqueira Trevisan ${ }^{1}$

1.Universidade Estadual de Campinas, Campinas (SP), Brazil.

*Corresponding author:vinisoubihe@uol.com.br

\section{BACKGROUND}

Chronic, recurrent, multifocal osteomyelitis (CRMO) is a rare, autoinflammatory bone disease, characterized by chronic, nonbacterial osteomyelitis, multifocal bone lesions, and multiple recurrence. Myositis is a rare clinical symptom of CRMO. Here, we report a case of $\mathrm{CRMO}$ with myositis of the right quadriceps femoris muscle.

\section{CASE REPORT}

A 36-year-old woman was admitted to our hospital with a 4-week history of pain and swelling of the anterior right tight. She had a medical history of CRMO, diagnosed at 2 years old, characterized by recurrent osteomyelitis affecting her vertebrae, mandible and long bones of the lower limbs, associated with juvenile idiopathic arthritis related to enthesitis, on current use of prednisone $10 \mathrm{mg} / \mathrm{day}$ and with previous multiple treatment regimens, including TNF- and IL-6 antagonists, suspended due to serious adverse events. Physical examination revealed a swollen anterior right, with one distal collection with spontaneous drainage of purulent secretion (Figure 1). Laboratory tests presented high levels of erythrocyte sedimentation rate $(101 \mathrm{~mm} / \mathrm{h})$ and C-reactive protein $(106 \mathrm{mg} / \mathrm{L})$ and normal creatine kinase level $(47 \mathrm{U} / \mathrm{L})$. Magnetic resonance imaging (MRI) revealed enhanced muscle lesion in the anterior compartment of the right tight compatible with chronic inflammatory muscular process with signs of current significant inflammatory activity (Figure 2). A bone scintigraphy revealed high uptake in the anterior compartment of the right thigh compatible with active myositis (Figure 3). A muscle biopsy of the right lateral vastus (Figure 4) showed nonspecific chronic inflammatory infiltration, distributed in multiple focuses with lymphoplasmacytic infiltrate with frequent eosinophils. Cultures resulted negative for bacterial, fungal and mycobacterial growth. From these characteristics, the diagnosis of myositis of the right quadriceps femoris was made. The patient was initially treated with ibuprofen and antibiotics until culture results were available, with no clinical improvement. Afterwards treatment with methotrexate and colchicine was started, showing improvement on clinical symptoms.

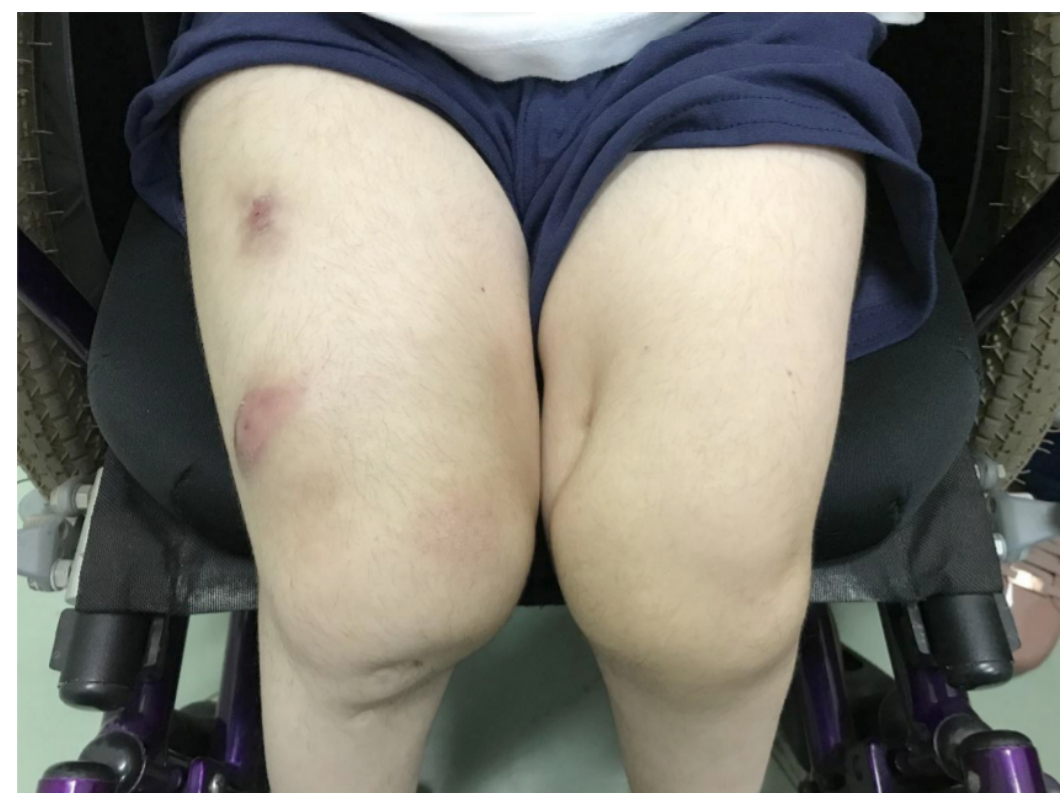

Figure 1. Physical exam findings in the patient.

Realização: 


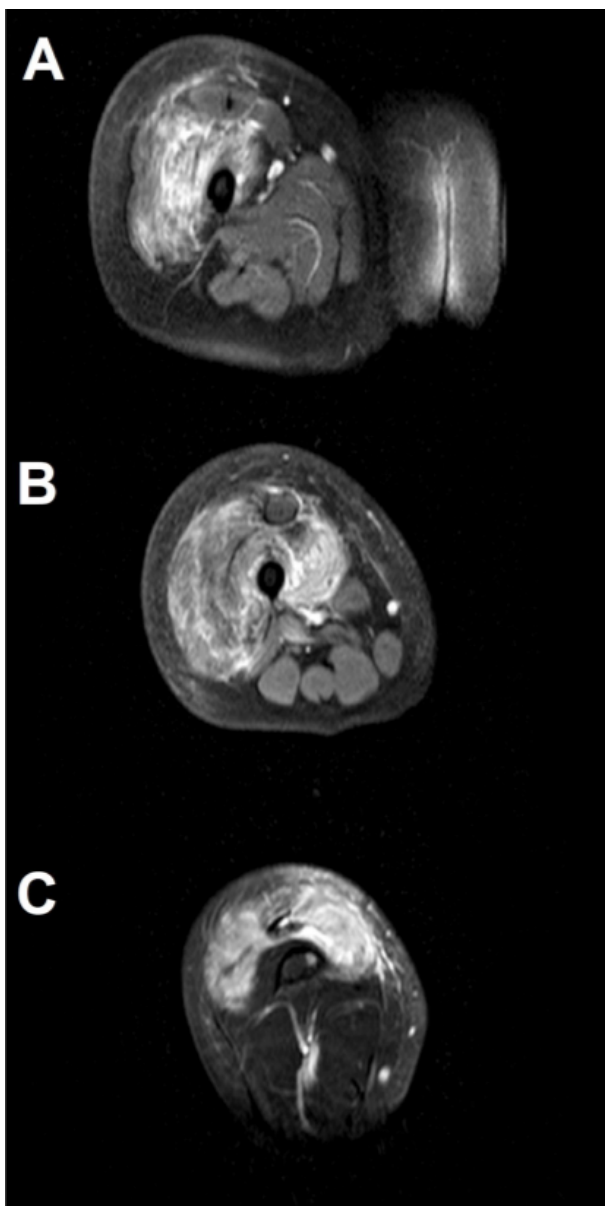

Figure 2. Magnetic resonance imaging (axial DP FAT SAT) shows higher enhanced intensity of the proximal (A), medium (B) and distal $(C)$ right femoris quadriceps muscle, suggesting myositis.
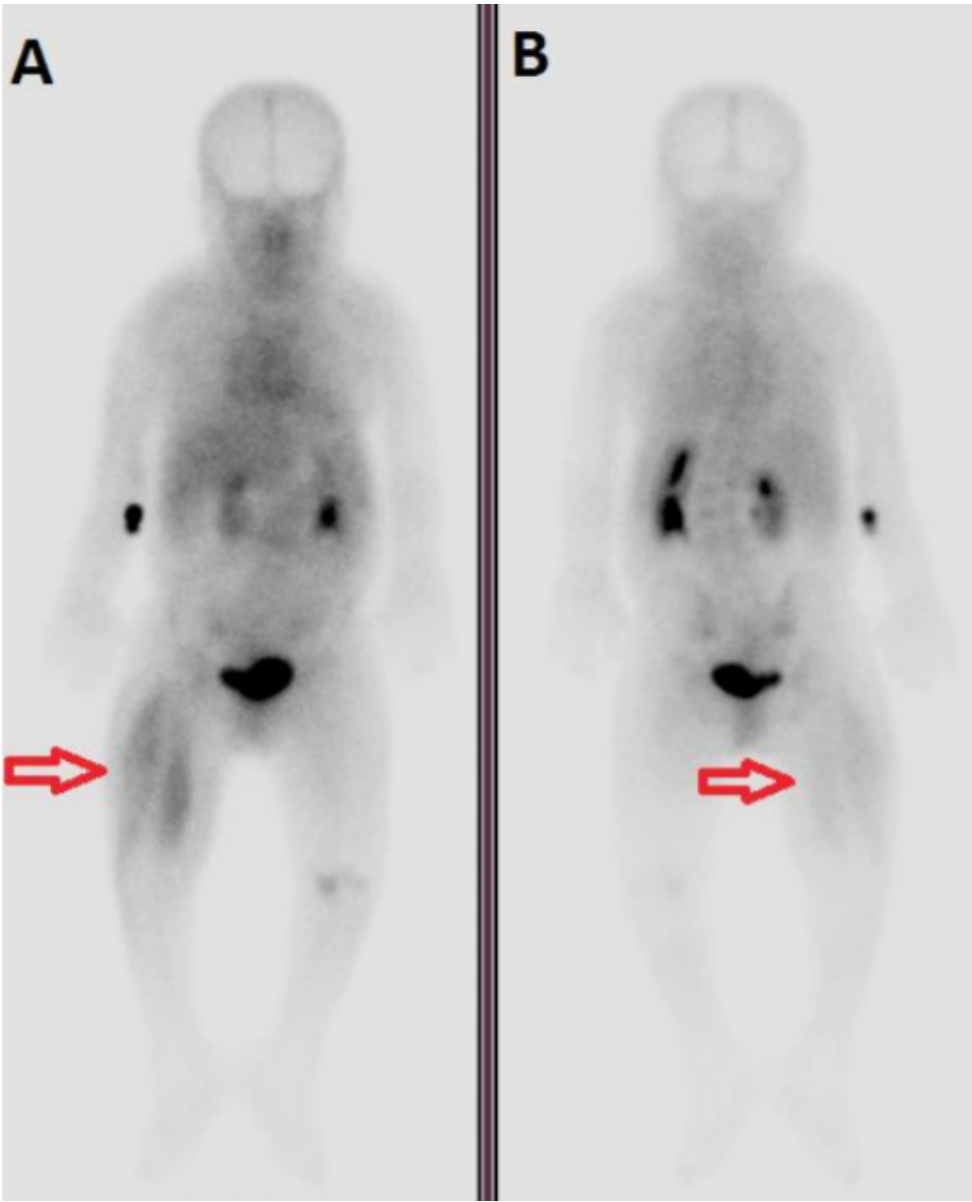

Figure 3. Bone scintigraphy, anterior (A) and posterior (B) projection, shows high uptake in the anterior compartment of the right thigh (red arrows) compatible with active myositis.
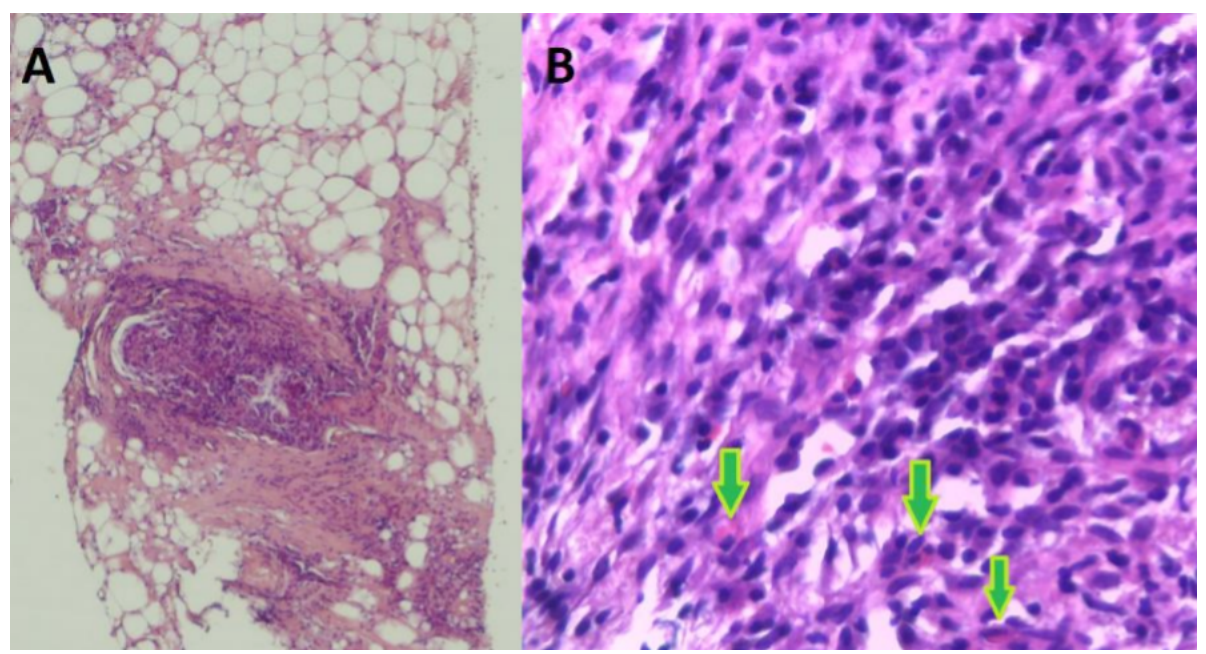

Figure 4. Muscle biopsy with histopathology findings of fibroadipose tissue with extensive stromal fibrosis (A) and multiple focuses of chronic inflammatory process (B) with lymphoplasmacytic infiltrate with eosinophils (green arrows). 


\section{CONCLUSION}

Myositis as a clinical symptom of CRMO is very rare, and only six cases have been previously reported. The muscles in the extremities were affected in most cases, and not necessarily in close to osteomyelitis. No patients showed muscle weakness. The elevation of creatine kinase levels was observed only in one patient. A muscle biopsy was performed in one patient and revealed interstitial myositis. Two patients were treated with tocilizumab, one patient with ibuprofen and pamidronate, one patient with colchicine. Physicians should be aware that myositis is rarely observed as a clinical symptom of CRMO.

\section{KEYWORDS:}

CRMO, Myositis, Autoinflammatory. 